\title{
Correlation between Serum Dehydroepiandrosterone Sulfate and LDL Cholesterol in Patients with Polycystic Ovarian Syndrome
}

\author{
Hantoro Gunawan ${ }^{1}$, Irvan Ipandii, Jusak Nugraha ${ }^{1}$, Ashon Sa'adi ${ }^{2}$ \\ ${ }^{1}$ Department of Clinical Pathology, Faculty of Medicine, Airlangga University/Dr. Soetomo Hospital, Surabaya, Indonesia. E-mail: \\ hantoro_md@yahoo.com \\ ${ }^{2}$ Department of Obstetrics and Gynecology, Faculty of Medicine, Airlangga University/Dr. Soetomo Hospital, Surabaya, Indonesia
}

\section{ABSTRACT}

Polycystic Ovarian Syndrome (PCOS) is the most prevalent endocrine disorder in the female of childbearing age. Polycystic ovarian syndrome patients are also at risk of metabolic and cardiovascular disorders. One of the factors that can affect those disorders is the androgen level. The adrenal gland produces dehydroepiandrosterone, and the effect of its metabolite dehydroepiandrosterone sulfate (DHEAS) is different from testosterone. The DHEA and DHEAS might have a protective effect on dyslipidemia and cardiovascular disorder. According to the National Cholesterol Education Program Adult Treatment Panel, Low-Density Lipoprotein (LDL), cholesterol is an important parameter for dyslipidemia therapy guidelines. This study aimed to analyze the correlation between DHEAS and LDL cholesterol. This research was a cross-sectional study. Dehydroepiandrosterone sulfate and LDL cholesterol levels were measured in 25 research subjects by chemiluminescent immunoassay (Immulite ${ }^{\circledR}$, Siemens) and colorimetric enzymatic assay (Dimension EXL ${ }^{\circ}$, Siemens). These subjects were divided into three groups, according to the Rotterdam 2003 criteria. Spearman and Pearson's correlation statistical analysis was used with a $p$-value $<0.05$. This study showed a moderate negative correlation between DHEAS and LDL cholesterol ( $r=-0.441, p=0.027$ ). Group D PCOS (non-hyperandrogenic) had a strong negative correlation between DHEAS and LDL cholesterol $(r=-0.717, p=0.001)$. Some references suggested that DHEA was shown to activate Peroxisome Proliferator-Activated Response y (PPAR Y) receptors, thereby increasing LDL cholesterol receptors in adipose tissue LDL cholesterol uptake from serum. The increase in DHEAS might indicate a better prognosis for PCOS patients in cardiovascular complications, especially in group D PCOS.

Keywords: Dehydroepiandrosterone sulfate, low-density lipoprotein cholesterol, polycystic ovarian syndrome

\section{INTRODUCTION}

Polycystic Ovarian Syndrome (PCOS) is the most prevalent endocrine disorder in the female of childbearing age. The prevalence of PCOS was approximately $4,5 \%$ in 2007 . $^{1}$ The rate increased to $8-10 \%$ because of the shift toward high calories diet and a sedentary lifestyle. ${ }^{1}$ The main complaint of PCOS was infertility and PCOS caused about $75 \%$ of infertility with anovulation. ${ }^{1}$ This problem would require patients to seek medical care infertility clinics.

Patients with PCOS were prone to other endocrine and metabolic abnormalities, mainly caused by insulin resistance and hyperandrogenemia. Insulin resistance and subsequent hyperinsulinemia caused an increase of Free Fatty Acid (FFA), which could induce cholesterol synthesis into Very-Low-Density Lipoprotein (VLDL) cholesterols and Low-Density Lipoprotein (LDL) cholesterols. Females with PCOS had four times greater risk of metabolic syndrome compared to the general population. ${ }^{2}$ Another research showed increasing evidence of stenotic coronary syndrome detected by angiography in females with PCOS. ${ }^{3}$

High androgen level was the critical factor of pathogenesis in PCOS. Hyperandrogenemia was associated with polycystic ovarium morphology and ovulatory dysfunction in PCOS patients and animal models. ${ }^{4}$ Androgen can be produced in the ovary or adrenal gland. The adrenal cortex exclusively produces dehydroepiandrosterone (DHEA) and its metabolite dehydroepiandrosterone sulfate (DHEAS). Some studies showed that around 20 to 50 percents of females with PCOS had high serum DHEAS. $^{4-6}$

Data about the effect of DHEAS on metabolic outcome remain contradictory. Previous research showed opposing effects of adrenal and ovarian androgen on body weight and lipid profile in females with PCOS. ${ }^{6-8}$ Köşüş et al. found a correlation between high DHEAS level and a good lipid profile. ${ }^{6}$ 
Chen et al. concluded that elevated serum DHEAS levels were associated with a lower risk of dyslipidemia, independent of testosterone level and insulin resistance. ${ }^{9}$ Brennan showed opposing effect of DHEAS and testosterone on metabolic complications in females with PCOS. ${ }^{10}$

Low-Density Lipoprotein Cholesterol (LDL-C) is the primary parameter for dyslipidemia therapeutic target according to National Cholesterol Education Program Adult Treatment Panel III (NCEP-ATP III). ${ }^{11}$ This parameter can represent the risk of dyslipidemia in patients with $P C O S$.

Research about DHEAS and PCOS in Indonesia is still limited. DHEAS might be a prognostic marker in females with PCOS, while the evidence was still lacking. This study aimed to analyze the correlation between DHEAS as adrenal androgen and LDL cholesterol in females with PCOS.

\section{METHODS}

The study was performed in March-December 2019, using a cross-sectional design and consecutive sampling. Subjects consisted of patients diagnosed according to Rotterdam 2003 criteria in various fertility centers, such as Ferina Hospital, Lombok 22 Hospital, Elizabeth Clinic, and Putri Hospital. All subjects were divided into four phenotypes according to the Rotterdam 2003 criteria. Anthropological parameters, serum DHEAS, and LDL cholesterol were measured on all subjects. Hyperandrogenic was determined using the Ferriman-Gallwey score of more than 8. Anovulation was determined using a prolonged menstrual cycle for more than 35 days. Polycystic ovarian morphology was determined using ultrasonography by a certified specialist, with ovarian volume $>10 \mathrm{~mm}^{3}$ ( $0.5 \mathrm{x}$ height $\mathrm{x}$ width $\mathrm{x}$ length). Patients were classified into Phenotype A if all three criteria were found (hyper androgen, anovulation, polycystic ovarian morphology). Patients were classified into Phenotype B if both hyperandrogenic and anovulation were found. Patients were classified into Phenotype $C$ if hyperandrogenism and polycystic ovarian morphology were found. Patients were classified into Phenotype $D$ if anovulation and polycystic ovarian morphology were found.

Laboratory tests were performed in the Clinical Pathology Laboratory, Dr. Soetomo Hospital, Surabaya. DHEAS levels were performed using the chemiluminescent immunoassay method with the Immulite-1000 ${ }^{\circledR}$ instrument (Siemens, Germany). Low-density lipoprotein cholesterol was measured using the enzymatic colorimetric method with the Dimension EXL $®$ instrument (Siemens, Germany).

Statistical analysis was performed using SPSS ver. 16.0. The correlation between DHEAS and LDL cholesterol was analyzed using Spearman test. The correlation between DHEAS and LDL cholesterol in each phenotype of PCOS was analyzed using the Spearman and Pearson test. The strength of correlation was determined using the coefficient of rho (r). P-value $<0.05$ was considered significant, with a confidence interval of $95 \%$.

Research permission was obtained from the Health Research Ethics Committee of the Universitas Airlangga, School of Medicine, with number 286/EC/KEPK/FKUA/2019.

\section{RESULTS AND DISCUSSION}

The results of this study revealed that 25 subjects were diagnosed with PCOS. The characteristics of the subjects were shown in Table 1. Patients with PCOS mostly sought medical care during their childbearing age because the main complaint was infertility. The

Table 1. Characteristics of subjects

\begin{tabular}{lccc}
\hline & $\mathbf{n}$ & Mean \pm SD & Median (min-max) \\
\hline Age $($ years) & 25 & $29.28 \pm 4.078$ & $29(24-39)$ \\
BMI $\left(\mathrm{kg} / \mathrm{m}^{2}\right)$ & 25 & $29.97 \pm 2.927$ & $30.1(19.6-35.5)$ \\
Serum DHEAS $(\mu \mathrm{g} / \mathrm{dL})$ & 25 & $697,60 \pm 272,243$ & $765(131-1000)$ \\
LDL cholesterol $(\mathrm{mg} / \mathrm{dL})$ & 25 & $88.68 \pm 28.939$ & $93(31-154)$ \\
Phenotype A* & 6 & - & - \\
Phenotype C & 1 & - & - \\
Phenotype D & 18 & - & - \\
\hline
\end{tabular}

Abbreviation: BMI, Body Mass Index; DHEAS, Dehydroepiandrosterone Sulfate; LDL: Low-Density Lipoprotein; *Phenotype according to The European Society for Human Reproduction and Embryology/American Society for Reproductive Medicine 2003 (Rotterdam criteria); Phenotype A, hyperandrogenic + anovulation+ polycystic ovarian morphology; Phenotype D, anovulation + polycystic ovarian morphology, $\mathrm{n}$ : number of subjects 
mean Body Mass Index (BMI) was $29.97 \mathrm{~kg} / \mathrm{m}^{2}$ (overweight). Previous research showed that PCOS patients were mostly obese. In contrast, other study showed that more PCOS subjects had normal BMI. ${ }^{5,12}$ Obesity might induce chronic hyperinsulinemia and insulin resistance, thus aggravating hormonal and metabolic disorders in patients with PCOS. More significant symptoms might be the reason underlying more overweight or obese subjects sought medical care in this study.

Wiweko found that phenotype $A$ and $D$ were the most prevalent phenotype in Indonesia. ${ }^{12}$ Huang and Yong from Singapore showed evidence of the unique characteristics of PCOS in the Asian population. The rate of polycystic ovarian morphology was higher, and hirsutism was lower in the Asian community than Caucasians. ${ }^{13}$ The Rotterdam 2003 criteria used the Ferriman-Gallwey score for hyperandrogenic determination, while this score was based on hirsutism. This score could be less sensitive in Asian PCOS patients. ${ }^{13}$ Higher incidence of polycystic ovarian morphology, and low sensitivity of Ferriman-Gallwey score in the Asian population might explain why phenotype $D$ was the most prevalent phenotype in this study.

There was no significant correlation between DHEAS and age in this study (Table 2). This result was following Mannic et al. ${ }^{8}$ This research subjects varied between 24 to 39 years, and their serum DHEAS levels remained high. Serum DHEAS declined after 40 years of age. Most PCOS patients who sought medical care were females at their childbearing age. Thus no significant correlation between a relatively high level of DHEAS and age might be found.

The moderate positive correlation between DHEAS and BMI was found in this study (Table 2). The result was similar to a previous study by Brennan et al. about the correlation between DHEAS dan insulin resistance in PCOS with $r=0.5(p<0.001){ }^{10}$ Rojas et al., from Venezuela found that obesity could increase visceral fat accumulation and cortisol metabolism. Higher cortisol metabolism causes hyperactivity of the hypothalamus-hypophysis-adrenal axis, increasing DHEAS synthesis and conversion to its metabolite
(DHEAS). ${ }^{14}$ Chen et al. in Taiwan found a different result that PCOS patients with high serum DHEAS had a lower risk of abdominal obesity. ${ }^{9}$ This finding might be caused by modulation of insulin growth factor binding protein 1 , thus inhibiting fat accumulation. Obesity, in some research, was not the causal factor of PCOS, but its presence alone would aggravate insulin resistance and hyperandrogenism in PCOS..$^{14,15}$

The correlation between DHEAS and LDL cholesterol was moderately negative, indicating that higher serum DHEAS will lower LDL cholesterol. DHEA can activate many receptors in adipose tissue. One of those receptors can start Peroxisome Proliferator-Activated Response $\gamma$ (PPAR $\gamma$ ). ${ }^{16}$ Activation of this receptor can upregulate the expression of LDL receptors in adipose tissue. The increase of $L D L$ receptors would increase the uptake of LDL cholesterol in blood vessels. This result was supported by an animal study performed by Henning et al. about the reduction of LDL cholesterol after DHEA supplementation. ${ }^{17}$ Lasco in Italy also found an $11.7 \%$ reduction of LDL cholesterol after 12 month DHEA supplementation on postmenopausal females. ${ }^{18}$ A study by Lerchbaum also found a negative correlation between DHEAS and LDL cholesterol. ${ }^{19}$

The low correlation between DHEAS and LDL cholesterol in classic PCOS phenotype A (Table 3) might be caused by increased other sources of androgen, such as testosterone. ${ }^{19}$ Sachdeva in India elevated testosterone and LDL cholesterol in phenotype A than different phenotypes. ${ }^{15}$ Testosterone has an opposite effect on DHEAS on LDL cholesterol. A strong correlation between DHEAS and LDL cholesterol in phenotype D showed that in this non-hyperandrogenic phenotype, no opposing androgen hormone would interfere with DHEAS function on LDL cholesterol.

Gambineri et al. found that PCOS patients with $11 \beta$-Hydroxysteroid dehydrogenase type 1 (11 $\beta$-HSD1) gene polymorphism showed elevated DHEAS and lower LDL cholesterol. That study supported the concept that there were different pathogenesis between each PCOS phenotypes. ${ }^{20}$

Table 2. Correlation between DHEAS, age, BMI, and LDL cholesterol

\begin{tabular}{lllcc}
\hline Correlation & with & $\mathbf{n}$ & $\mathbf{r}_{\mathbf{s}}$ & P-value \\
\hline \multirow{2}{*}{ DHEAS } & Age & 25 & -0.016 & 0.941 \\
& BMI & 25 & 0.533 & 0.006 \\
& LDL cholesterol & 25 & -0.441 & 0.027 \\
\hline
\end{tabular}

Abbreviation: DHEAS, Dehydroepiandrosterone Sulfate; LDL, Low-Density Lipoprotein; n, number of subjects; BMI, Body Mass Index 
Table 3. Correlation between DHEAS and LDL in each PCOS phenotype

\begin{tabular}{lccc}
\hline Phenotype* $^{*}$ & $\mathbf{n}$ & $\mathbf{r} / \mathbf{r}_{\mathbf{s}}$ & P-value \\
\hline A & 6 & -0.030 & 0.954 \\
D & 18 & -0.717 & 0.001 \\
\hline
\end{tabular}

Abbreviation: *Phenotype according to The European Society for Human Reproduction and Embryology/American Society for Reproductive Medicine 2003 (Rotterdam criteria); Phenotype A, hyperandrogenic+anovulation+polycystic ovary morphology; Phenotype $D$, anovulation+polycystic ovarian morphology; $n$, number of case

Table 4 showed a significant difference between hyperandrogenic determination using clinical hirsutism (Ferriman-Gallwey score) and DHEAS ( $p<0.001)$. Fourteen subjects $(77.8 \%)$ that were previously determined as phenotype $D$ could be reclassified to phenotype $A$ with the DHEAS test's aid. Huang and Yong found that PCOS patients in the Asian population were less hairy than Caucasian. Thus Ferriman-Gallwey score would be less sensitive. ${ }^{13}$ Examination of androgen, like testosterone or DHEAS in Asian PCOS patients, might help determine biochemical hyperandrogenic.

Table 4. Determination of hyperandrogen with DHEAS

\begin{tabular}{lccc}
\hline Phenotype* $^{*}$ & $\begin{array}{c}\text { n without } \\
\text { DHEAS }\end{array}$ & $\begin{array}{c}\text { with } \\
\text { DHEAS }\end{array}$ & P-value \\
\hline A & 6 & 20 & 0.001 \\
D & 18 & 4 & \\
\hline
\end{tabular}

Abbreviation: *Phenotype according to The European Society for Human Reproduction and Embryology/American Society for Reproductive Medicine 2003 (Rotterdam criteria); Phenotype A, hyperandrogenic+anovulation+polycystic ovary morphology; Phenotype D, anovulation+polycystic ovary morphology; $n$, number of the case; DHEAS, Dehydroepiandrosterone Sulfate

\section{CONCLUSION AND SUGGESTION}

Adrenal hyperandrogenism might contribute to a better prognosis in PCOS patients. The measurement of DHEAS levels can help further classify a high metabolic and cardiovascular risk in those patients; thus, better prevention can be initiated. Further experimental research to determine the relationship between dyslipidemia and DHEAS might be needed.

\section{REFERENCES}

1. Santoso B. Sindroma ovarium polikistik: Problem reproduksi dan tantangannya terkait gaya hidup. Unair repository. Surabaya, Airlangga University Press, 2020. http://repository.unair.ac.id/40072/1/ gdlhub-gdl-grey-2016-santosobud-40453-pg.12-14-s.pdf (accessed 14 December, 2019).
2. Cussons AJ, Watts GF, Burke V, Shaw JE, Zimmet PZ, Stuckey B. Cardiometabolic risk in polycystic ovary syndrome: A comparison of different approaches to defining the metabolic syndrome. Human Reproduction, 2008; 23(10): 2352-2358.

3. Rizzo M. Long-term consequences of polycystic ovary syndrome on cardiovascular risk. Fertility and Sterility, 2009; 91(4): 1563-1567.

4. Wild RA, Rizzo M, Clifton S, Carmina E. Lipid levels in polycystic ovary syndrome: Systematic review and meta-analysis. Fertility and Sterility, 2011; 95(3): 1073-1079.

5. Christodoulaki C, Pergialiotis V. Dehydroepiandrosterone-sulfate, insulin resistance, and ovarian volume estimation in patients with polycystic ovarian syndrome. Journal of Family and Reproductive Health, 2017; 11(1): 24-29.

6. Köşüş N, Köşüş $A$, Kamalak $Z$, Hizli $D$, Turhan NÖ. Impact of adrenal versus ovarian androgen ratio on signs and symptoms of polycystic ovarian syndrome. Gynecological Endocrinology, 2012; 28(8): 611-614.

7. Simoncini T, Mannella P, Fornari L, Varone G, Caruso A, et al. Dehydroepiandrosterone modulates endothelial nitric oxide synthesis via direct genomic and nongenomic mechanisms. Endocrinology, 2003; 144(8): 3449-3455.

8. Mannic T, Viguie J, Rossier MF. In-vivo and in-vitro evidences of dehydroepiandrosterone protective role on the cardiovascular system. International Journal of Endocrinology and Metabolism, 2015; 13(2): 1-5.

9. Chen $M$, Chen $C$, Yang J, Chen $C$, Ho H, et al. High serum dehydroepiandrosterone sulfate is associated with phenotypic acne and a reduced risk of abdominal obesity in women with polycystic ovary syndrome. Hum. Reprod, 2011; 26: 219-220.

10. Brennan K, Huang A, Azziz R. Dehydroepiandrosterone sulfate and insulin resistance in patients with polycystic ovary syndrome. Fertility and Sterility, 2009; 91(5): 1848-1852.

11. Grundy S, Berker D, Clark L, Cooper R. Third Report of the National Cholesterol Education Program (NCEP) Expert panel on detection, evaluation, and treatment of high blood cholesterol in adults (adult treatment panel III). NIH Publication, 2002; 2: 3-4.

12. Wiweko $B$, Indra $I$, Susanto $C$, Natadisastra $M$, Hestiantoro A. The correlation between serum AMH and HOMA IR among PCOS phenotypes. BMC Research Notes, 2018; 1: 1-6.

13. Huang Z, Yong E. Ethnic differences: Is there an Asian phenotype for polycystic ovarian syndrome?. Best Practice \& Research Clinical Obstetrics \& Gynaecology, 2016; 2: 1-28.

14. Rojas J, Chávez M, Olivar L, Rojas M, Morillo J, et al. Polycystic ovary syndrome, insulin resistance, and obesity: Navigating the pathophysiologic labyrinth. International Journal of Reproductive Medicine, 2014; 14:1-17.

15. Sachdeva G, Gainder S, Suri V, Sachdeva N, Chopra S. Comparison of the different PCOS phenotypes based on clinical metabolic, and hormonal profile, and their 
response to clomiphene. Indian J Endocr and Metab, 2019; 23: 326-331.

16. Chui, PC, Guan H, Lehrke M, Lazar MA. PPAR $\gamma$ regulates adipocyte cholesterol metabolism via oxidized LDL receptor 1 . Journal of Clinical Investigation, 2005; 115(8): 2244-2256.

17. Hennings C, Kurzman I, Haffa A, Kemnitz J, Macewen E. The effect of high-fat diet and dehydroepiandrosterone (DHEA) administration in the rhesus monkey. In-Vivo, 1995; 5: 415-420.

18. Lasco A, Frisina N, Morabito N, Gaudio A, Morini E, et al. Metabolic effects of dehydroepiandrosterone replacement therapy in post-menopausal women.
European Journal of Endocrinology, 2001; 145: 457-461. 19. Lerchbaum E, Schwetz V, Giuliani A, Pieber TR. Opposing effects of dehydroepiandrosterone sulfate and free testosterone on metabolic phenotype in women with polycystic ovary syndrome. Fertility and Sterility, 2012; 98(5): 1318-1325.e1.

20. Gambineri A, Vicennati V, Genghini S, Tomassoni F, Pagotto $U$, et al. Genetic variation in 11 B-hydroxysteroid dehydrogenase type 1 predicts adrenal hyperandrogenism among lean women with polycystic ovary syndrome. The Journal of Clinical Endocrinology \& Metabolism, 2006; 91(6): 2295-23. 